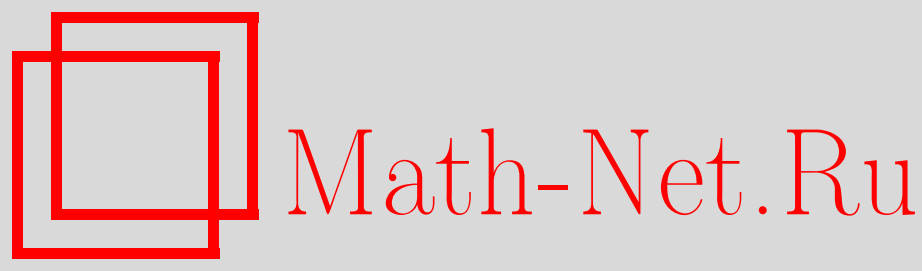

А. В. Разумов, Ю. Г. Строганов, Статистическая модель трех цветов с граничными условиями типа доменной стенки. Тригонометрический предел, ТМФ, 2009, том 161, номер 2, 154-163

DOI: https://doi.org/10.4213/tmf6428

Использование Общероссийского математического портала Math-Net.Ru подразумевает, что вы прочитали и согласны с пользовательским соглашением http: //www . mathnet.ru/rus/agreement

Параметры загрузки:

IP : 54.197 .130 .99

26 апреля 2023 г., 13:50:01

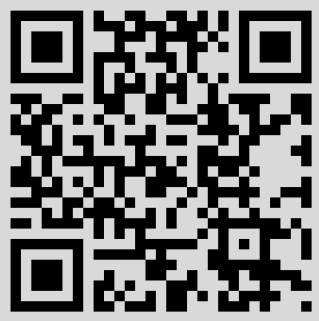




\section{СТАТИСТИЧЕСКАЯ МОДЕЛЬ ТРЕХ ЦВЕТОВ С ГРАНИЧНЫМИ УСЛОВИЯМИ ТИПА ДОМЕННОЙ СТЕНКИ. ТРИГОНОМЕТРИЧЕСКИЙ ПРЕДЕЛ}

Рассмотрен нетривиальный тригонометрический предел статистической модели трех цветов с граничными условиями типа доменной стенки. В этом пределе решены функциональные уравнения, построенные в предыдущей работе авторов, и найдены новые детерминантные представления для частичных статистических сумм.

Ключевые слова: модель трех цветов, статистическая сумма, тригонометрический предел.

\section{1. ВВЕДЕНИЕ}

В настоящей работе мы продолжаем исследование статистической модели трех цветов с граничными условиями типа доменной стенки, начатое в работе [1]. Рассматриваемая модель непосредственно связана с моделью трех цветов Бакстеpa [2], [3]. Сначала напомним необходимые определения и факты.

Состояния модели задаются различными раскрасками тремя цветами граней квадратной решетки размера $(n+1) \times(n+1)$. При этом допустимыми считаются только те раскраски, для которых любые две смежные грани имеют разный цвет. Удобно маркировать цвета элементами кольца $\mathbb{Z}_{3}$. Мы обозначаем элементы $\mathbb{Z}_{3}$ как $\overline{0}, \overline{1}$ и $\overline{2}$. Когда элемент $\mathbb{Z}_{3}$ появляется там, где должно стоять целое число, то он рассматривается как соответствующее число 0, 1 или 2. Наоборот, когда целое число $i$ стоит там, где должен стоять элемент $\mathbb{Z}_{3}$, оно рассматривается как элемент $\mathbb{Z}_{3}$, соответствующий остатку от деления $i$ на 3 . Ясно, что цвета смежных граней различны тогда и только тогда, когда соответствующие элементы $\mathbb{Z}_{3}$ отличаются на $+\overline{1}$ или $-\overline{1}$. Граничные условия типа доменной стенки означают, что при движении от любой граничной грани против часовой стрелки вдоль границы цвет меняется на $+\overline{1}$ от грани к грани для вертикальных границ и на $-\overline{1}$ для горизонтальных границ. Пример таких условий изображен на рис. 1.

Больцмановский вес состояния является произведением больцмановских весов внутренних вершин решетки, а сумма весов всех возможных состояний является статистической суммой модели. Мы предполагаем, что больцмановский вес вершины

* Институт физики высоких энергий, Протвино, Московская обл., Россия. E-mail: yuri.stroganov@ihep.ru 


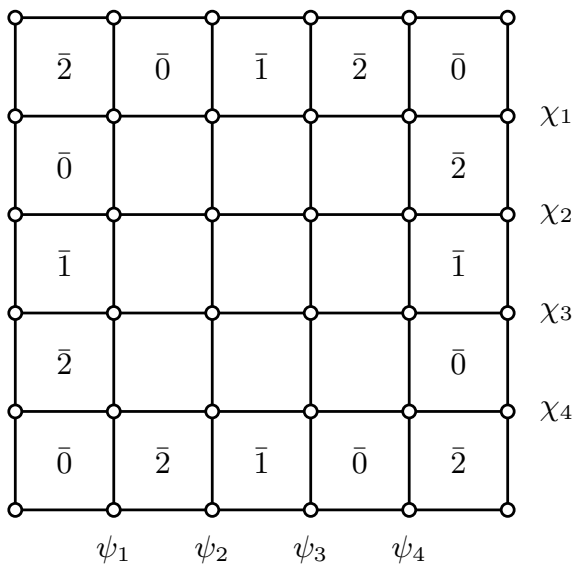

Рис. 1
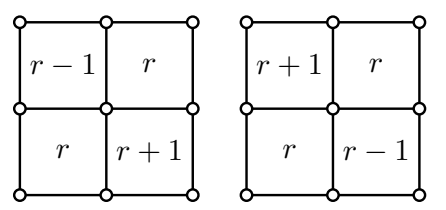

$\alpha_{r}^{\prime}(\varphi)$

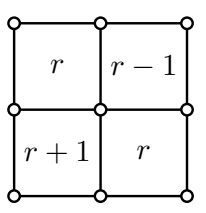

$\beta_{r}(\varphi)$

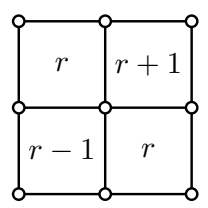

$\beta_{r}^{\prime}(\varphi)$

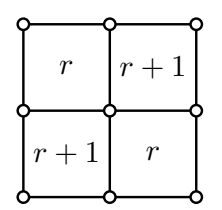

$\gamma_{r}(\varphi)$

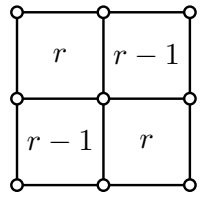

$\gamma_{r}^{\prime}(\varphi)$

Рис. 2

определяется цветами четырех смежных граней. Чтобы получить интегрируемую систему, мы предполагаем, что он зависит также от спектрального параметра так, что выполняются соотношения звезда-треугольник (уравнения Янга-Бакстера). Нетрудно убедиться в том, что имеется шесть типов возможных вершинных цветовых конфигураций, приведенных на рис. 2 , где $r$ - один из цветов $\overline{0}, \overline{1}$ или $\overline{2}$. Мы будем использовать веса, найденные в работе [4], которые имеют вид

$$
\begin{gathered}
\alpha_{r}(\varphi)=\alpha_{r}^{\prime}(\varphi)=\frac{\theta_{1}(\pi / 3-\varphi)}{\theta_{1}(2 \pi / 3)}, \\
\beta_{r}(\varphi)=\beta_{r}^{\prime}(\varphi)=\zeta_{r}^{1 / 2} \frac{\theta_{1}(\pi / 3+\varphi)}{\theta_{1}(2 \pi / 3)}, \\
\gamma_{r}(\varphi)=\frac{\theta_{4}(\lambda+2 \pi(r+1 / 2) / 3+\varphi)}{\theta_{4}(\lambda+2 \pi r / 3)}, \quad \gamma_{r}^{\prime}(\varphi)=\frac{\theta_{4}(\lambda+2 \pi(r-1 / 2) / 3-\varphi)}{\theta_{4}(\lambda+2 \pi r / 3)},
\end{gathered}
$$

где $\theta_{1}$ и $\theta_{4}$ - стандартные эллиптические $\theta$-функции модуля $p=e^{i \pi \tau}[5], \varphi-$ спектральный параметр, $\lambda$ - некоторый фиксированный параметр,

$$
\zeta_{r}=\frac{\theta_{4}(\lambda+2 \pi(r-1) / 3) \theta_{4}(\lambda+2 \pi(r+1) / 3)}{\theta_{4}^{2}(\lambda+2 \pi r / 3)} .
$$

Точнее, веса (1) связаны с весами, найденными в [4], калибровочным преобразованием. В работе [1] мы помечали эти веса и соответствующую статистическую сумму 
тильдой. Для упрощения обозначений в настоящей работе мы опускаем соответствующие тильды.

Рассмотрим неоднородный случай, когда внутренние горизонтальные линии маркируются переменными $\chi_{i}, i=1, \ldots, n$, а внутренние вертикальные линии - переменными $\psi_{i}, i=1, \ldots, n$ (см. рис. 1). С вершиной, находящейся на пересечении линии, помеченной переменной $\chi_{i}$, и линии, помеченной переменной $\psi_{j}$, мы связываем спектральный параметр $\chi_{i}-\psi_{j}$. Полная статистическая сумма $Z_{n}(\{\chi\} ;\{\psi\})$ является суммой частичных статистических сумм:

$$
Z_{n}(\{\chi\} ;\{\psi\})=\sum_{r \in \mathbb{Z}_{3}} Z_{n}^{r}(\{\chi\} ;\{\psi\})
$$

где $r$ - цвет левой верхней грани решетки.

Введем следующие функции:

$$
\begin{aligned}
F_{n}^{r}(\{\chi\} ;\{\psi\})= & \frac{1}{\theta_{4}(\lambda+2 \pi(r+n) / 3)} \times \\
& \times \prod_{\substack{i, j=1 \\
i<j}}^{n} \theta_{1}\left(\chi_{i}-\chi_{j}\right) \prod_{i, j=1}^{n} \theta_{1}\left(\chi_{i}-\psi_{j}\right) \prod_{\substack{i, j=1 \\
i<j}}^{n} \theta_{1}\left(\psi_{i}-\psi_{j}\right) Z_{n}^{r}(\{\chi\} ;\{\psi\}) .
\end{aligned}
$$

В работе [1] было доказано, что функции $F_{n}^{r}(\{\chi\} ;\{\psi\})$ удовлетворяют функциональным уравнениям

$$
\begin{aligned}
& \sum_{s \in \mathbb{Z}_{3}} F_{n}^{r+s}\left(\chi_{1}, \ldots, \chi_{k}+2 \pi s / 3, \ldots, \chi_{n} ;\{\psi\}\right)=0 \\
& \sum_{s \in \mathbb{Z}_{3}} F_{n}^{r+s}\left(\{\chi\} ; \psi_{1}, \ldots, \psi_{k}-2 \pi s / 3, \ldots, \psi_{n}\right)=0 .
\end{aligned}
$$

Эти уравнения аналогичны уравнениям, полученным Строгановым [6] для шестивершинной модели с граничными условиями типа доменной стенки для специального значения кроссинг-параметра $\eta=2 \pi / 3$. Они оказываются очень полезными при решении задач перечисления матриц чередующихся знаков [6] (см. также статьи [7]-[9]).

В настоящей работе мы решаем уравнения (2) и (3) в тригонометрическом пределе и находим детерминантное представление для частичных статистических сумм. Заметим, что статистическая модель трех цветов является частным случаем восьмивершинной модели SOS, предложенной в статье [10]. Детерминантное представление статистической суммы этой модели для случая граничных условий типа доменной стенки было получено Розенгреном [11]. Наше представление может быть получено из представления Розенгрена, если использовать результаты о детерминантах из работ [12], [13]. Однако мы предпочитаем использовать функциональные уравнения в надежде, что такой подход окажется полезным для исследования общего эллиптического случая. 


\section{2. ТРИГОНОМЕТРИЧЕСКИЙ ПРЕДЕЛ}

Если $\operatorname{Im} \tau \rightarrow \infty$ при $p \rightarrow 0$, то веса (1) принимают вид

$$
\begin{gathered}
\alpha_{r}(\varphi)=\alpha_{r}^{\prime}(\varphi)=\frac{\sin (\pi / 3-\varphi)}{\sin (2 \pi / 3)}, \quad \beta_{r}(\varphi)=\beta_{r}^{\prime}(\varphi)=\frac{\sin (\pi / 3+\varphi)}{\sin (2 \pi / 3)}, \\
\gamma_{r}(\varphi)=\gamma_{r}^{\prime}(\varphi)=1,
\end{gathered}
$$

так как при $p \rightarrow 0$

$$
\theta_{1}(\varphi \mid p)=2 p^{1 / 4} \sin \varphi+O\left(p^{9 / 4}\right), \quad \theta_{4}(\varphi \mid p)=1+O(p) .
$$

Таким образом, мы получаем веса шестивершинной модели при специальном значении кроссинг-параметра $\eta=2 \pi / 3$. В этом случае каждая частичная статистическая сумма модели трех цветов совпадает со статистической суммой шестивершинной модели для этого значения кроссинг-параметра.

Существует также нетривиальный тригонометрический предел. Для того чтобы его получить, сделаем подстановку $\lambda \rightarrow \lambda+\pi \tau / 2$ и используем соотношение

$$
\theta_{4}(\varphi \mid p)=i p^{1 / 4} e^{-i \varphi} \theta_{1}(\varphi-\pi \tau / 2 \mid p)
$$

Веса (1) принимают теперь вид

$$
\begin{aligned}
& \alpha_{r}(\varphi)=\alpha_{r}^{\prime}(\varphi)=\frac{\theta_{1}(\pi / 3-\varphi)}{\theta_{1}(2 \pi / 3)}, \\
& \beta_{r}(\varphi)=\beta_{r}^{\prime}(\varphi)=\zeta_{r}^{1 / 2} \frac{\theta_{1}(\pi / 3+\varphi)}{\theta_{1}(2 \pi / 3)}, \\
& \gamma_{r}(\varphi)=e^{-i(\pi / 3+\varphi)} \frac{\theta_{1}(\lambda+2 \pi(r+1 / 2) / 3+\varphi)}{\theta_{1}(\lambda+2 \pi r / 3)}, \\
& \gamma_{r}^{\prime}(\varphi)=e^{i(\pi / 3+\varphi)} \frac{\theta_{1}(\lambda+2 \pi(r-1 / 2) / 3-\varphi)}{\theta_{1}(\lambda+2 \pi r / 3)},
\end{aligned}
$$

где

$$
\zeta_{r}=\frac{\theta_{1}(\lambda+2 \pi(r-1) / 3) \theta_{1}(\lambda+2 \pi(r+1) / 3)}{\theta_{1}^{2}(\lambda+2 \pi r / 3)} .
$$

Мы будем работать с полиномами вместо тригонометрических функций, поэтому введем следующие обозначения:

$$
w=e^{i \varphi}, \quad a=e^{i \pi / 3}, \quad b=e^{i \lambda}, \quad \sigma(w)=w-w^{-1} .
$$

Тогда при $p \rightarrow 0$ получаем

$$
\begin{array}{ll}
\alpha_{r}(w)=\alpha_{r}^{\prime}(w)=\frac{\sigma\left(a w^{-1}\right)}{\sigma\left(a^{2}\right)}, & \beta_{r}(u)=\beta_{r}^{\prime}(u)=\zeta_{r}^{1 / 2} \frac{\sigma(a w)}{\sigma\left(a^{2}\right)}, \\
\gamma_{r}(w)=a^{-1} w^{-1} \frac{\sigma\left(a^{2 r+1} b w\right)}{\sigma\left(a^{2 r} b\right)}, & \gamma_{r}^{\prime}(u)=a w \frac{\sigma\left(a^{2 r-1} b w^{-1}\right)}{\sigma\left(a^{2 r} b\right)}
\end{array}
$$


где

$$
\zeta_{r}=\frac{\sigma\left(a^{2(r-1)} b\right) \sigma\left(a^{2(r+1)} b\right)}{\sigma^{2}\left(a^{2 r} b\right)} .
$$

Прежде чем перейти к тригонометрическому пределу для функций $F_{n}^{r}(\{x\} ;\{y\})$, разделим их на $\left(-i p^{1 / 4}\right)^{n(2 n-1)}$, сохраняя прежние обозначения. Нетрудно видеть, что при $p \rightarrow 0$ мы имеем

$$
F_{n}^{r}(\{x\} ;\{y\})=\frac{a^{2(r+n)} b}{\sigma\left(a^{2(r+n)} b\right)} \prod_{\substack{i, j=1 \\ i<j}}^{n} \sigma\left(x_{i} x_{j}^{-1}\right) \prod_{i, j=1}^{n} \sigma\left(x_{i} y_{j}^{-1}\right) \prod_{\substack{i, j=1 \\ i<j}}^{n} \sigma\left(y_{i} y_{j}^{-1}\right) Z_{n}^{r}(\{x\} ;\{y\}),
$$

где $x_{i}=e^{i \chi_{i}}, y_{i}=e^{i \psi_{i}}$. Теперь функциональные уравнения (2) и (3) могут быть переписаны в виде ${ }^{1)}$

$$
\begin{gathered}
\sum_{s \in \mathbb{Z}_{3}} F_{n}^{r+s}\left(x_{1}, \ldots, a^{2 s} x_{k}, \ldots, x_{n} ;\{y\}\right)=0, \\
\sum_{s \in \mathbb{Z}_{3}}^{2} F_{n}^{r+s}\left(\{x\} ; y_{1}, \ldots, a^{-2 s} y_{k}, \ldots, y_{n}\right)=0 .
\end{gathered}
$$

В разделе 3 мы решим эти уравнения.

\section{3. РЕШЕНИЕ ФУНКЦИОНАЛЬНЫХ УРАВНЕНИЙ}

При фиксированном $n$ в каждое из функциональных уравнений (6) и (7) входят все три функции $F_{n}^{s}(\{x\} ;\{y\})$. Удобно ввести другой набор функций и переписать функциональные уравнения так, чтобы в каждое функциональное уравнение входила только одна новая функция. Для этого выполним дискретное преобразование Фурье:

$$
W_{n}^{r}(\{x\} ;\{y\})=\sum_{s \in \mathbb{Z}_{3}} a^{-2 r s} F_{n}^{s}(\{x\} ;\{y\}),
$$

обращение к которому дается равенством

$$
F_{n}^{r}(\{x\} ;\{y\})=\frac{1}{3} \sum_{s \in \mathbb{Z}_{3}} a^{2 r s} W_{n}^{s}(\{x\} ;\{y\}) .
$$

В терминах функций $W_{n}^{s}(\{x\} ;\{y\})$ функциональные уравнения (6) и (7) принимают вид

$$
\begin{gathered}
\sum_{s \in \mathbb{Z}_{3}} a^{2 r s} W_{n}^{r}\left(x_{1}, \ldots, a^{2 s} x_{k}, \ldots, x_{n} ;\{y\}\right)=0 \\
\sum_{s \in \mathbb{Z}_{3}} a^{2 r s} W_{n}^{r}\left(\{x\} ; y_{1}, \ldots, a^{-2 s} y_{k}, \ldots, y_{n}\right)=0 .
\end{gathered}
$$

Учитывая соответствие между состояниями модели трех цветов и шестивершинной модели, найденное Ленардом [14], мы приходим к выводу, что в любом состоянии

\footnotetext{
1) $\mathrm{B}$ соответствии с нашими соглашениями $a^{ \pm 2 \bar{m}}=a^{ \pm 2 m}$.
} 
модели трех цветов в каждом столбце вершин есть по крайней мере одна вершина типа $\gamma$, и число вершин типа $\gamma$ всегда на единицу больше, чем число вершин типа $\gamma^{\prime}$. Используя явный вид весов в тригонометрическом пределе (4), (5), мы видим, что

$$
\begin{gathered}
W_{n}^{r}\left(x_{1}, \ldots,-x_{k}, \ldots, x_{n} ;\{y\}\right)=(-1)^{n} W_{n}^{r}\left(x_{1}, \ldots, x_{k}, \ldots, x_{n} ;\{y\}\right), \\
W_{n}^{r}\left(\{x\} ; y_{1}, \ldots,-y_{k}, \ldots, y_{n}\right)=(-1)^{n} W_{n}^{r}\left(\{x\} ; y_{1}, \ldots, y_{k}, \ldots, y_{n}\right) .
\end{gathered}
$$

Тогда

$$
\begin{aligned}
W_{n}^{r}\left(x_{1}, \ldots, x_{k}, \ldots, x_{n} ;\{y\}\right) & =\sum_{\ell=1}^{3 n} \alpha_{n, k, l}^{r}\left(x_{1}, \ldots, \hat{x}_{k}, \ldots, x_{n} ;\{y\}\right) x_{k}^{3 n-2 \ell}, \\
W_{n}^{r}\left(\{x\} ; y_{1}, \ldots, y_{k}, \ldots, y_{n}\right) & =\sum_{\ell=0}^{3 n-1} \beta_{n, k, l}^{r}\left(\{x\} ; y_{1}, \ldots, \hat{y}_{k}, \ldots, y_{n}\right) y_{k}^{3 n-2 \ell},
\end{aligned}
$$

где шляпка означает пропуск соответствующего аргумента.

Вид функциональных уравнений (8) и (9) подсказывает введение функций

$$
V_{n}^{r}(\{x\} ;\{y\})=\prod_{i=1}^{n}\left(x_{i}^{r} y_{i}^{-r}\right) W_{n}^{r}(\{x\} ;\{y\}),
$$

которые удовлетворяют более простым функциональным уравнениям

$$
\begin{aligned}
& \sum_{s \in \mathbb{Z}_{3}} V_{n}^{r}\left(x_{1}, \ldots, a^{2 s} x_{k}, \ldots, x_{n} ;\{y\}\right)=0 \\
& \sum_{s \in \mathbb{Z}_{3}} V_{n}^{r}\left(\{x\} ; y_{1}, \ldots, a^{2 s} y_{k}, \ldots, y_{n}\right)=0 .
\end{aligned}
$$

В итоге вместо соотношений (10) и (11) мы имеем соотношения

$$
\begin{gathered}
V_{n}^{r}\left(x_{1}, \ldots,-x_{k}, \ldots, x_{n} ;\{y\}\right)=(-1)^{3 n+r} V_{n}^{r}\left(x_{1}, \ldots, x_{k}, \ldots, x_{n} ;\{y\}\right), \\
V_{n}^{r}\left(\{x\} ; y_{1}, \ldots,-y_{k}, \ldots, y_{n}\right)=(-1)^{3 n-r} V_{n}^{r}\left(\{x\} ; y_{1}, \ldots, y_{k}, \ldots, y_{n}\right),
\end{gathered}
$$

а вместо представлений (12), (13) - представления

$$
\begin{aligned}
V_{n}^{r}\left(x_{1}, \ldots, x_{k}, \ldots, x_{n} ;\{y\}\right) & =\sum_{\ell=1}^{3 n} \gamma_{n, k, l}^{r}\left(x_{1}, \ldots, \hat{x}_{k}, \ldots, x_{n} ;\{y\}\right) x_{k}^{3 n-2 \ell+r} \\
V_{n}^{r}\left(\{x\} ; y_{1}, \ldots, y_{k}, \ldots, y_{n}\right) & =\sum_{\ell=0}^{3 n-1} \delta_{n, k, l}^{r}\left(\{x\} ; y_{1}, \ldots, \hat{y}_{k}, \ldots, y_{n}\right) y_{k}^{3 n-2 \ell-r} .
\end{aligned}
$$

Рассмотрим свойства функций $V_{n}^{r}(\{x\} ;\{y\})$ для разных значений $r$. Пусть $r=\overline{0}$. В этом случае функциональные уравнения (14), (15) дают

$$
\begin{aligned}
V_{n}^{\overline{0}}\left(x_{1}, \ldots, x_{k}, \ldots, x_{n} ;\{y\}\right) & =\sum_{\substack{\ell=1 \\
3 \nmid \ell}}^{3 n-1} \gamma_{n, k, l}^{\overline{0}}\left(x_{1}, \ldots, \hat{x}_{k}, \ldots, x_{n} ;\{y\}\right) x_{k}^{3 n-2 \ell}, \\
V_{n}^{\overline{0}}\left(\{y\} ; y_{1}, \ldots, y_{k}, \ldots, y_{n}\right) & =\sum_{\substack{\ell=1 \\
3 \nmid \ell}}^{3 n-1} \delta_{n, k, l}^{\overline{0}}\left(\{x\} ; y_{1}, \ldots, \hat{y}_{k}, \ldots, y_{n}\right) y_{k}^{3 n-2 \ell} .
\end{aligned}
$$


Введем обозначения

$$
u_{2 i-1}=x_{i}, \quad u_{2 i}=y_{i}, \quad i=1, \ldots, n,
$$

и сформулируем необходимые нам свойства функций $V_{n}^{\overline{0}}(\{u\})$.

1. Для каждого $\mu=1, \ldots, 2 n$ функция $V_{n}^{\overline{0}}(\{u\})$ удовлетворяет функциональному уравнению

$$
\begin{gathered}
V_{n}^{\overline{0}}\left(u_{1}, \ldots, u_{\mu}, \ldots, u_{2 n}\right)+V_{n}^{\overline{0}}\left(u_{1}, \ldots, a^{2} u_{\mu}, \ldots, u_{2 n}\right)+ \\
+V_{n}^{\overline{0}}\left(u_{1}, \ldots, a^{4} u_{\mu}, \ldots, u_{2 n}\right)=0 .
\end{gathered}
$$

2. Для каждого $\mu=1, \ldots, 2 n$ функция $u_{\mu}^{3 n-2} V_{n}^{\overline{0}}(\{u\})$ является полиномом по $u_{\mu}^{2}$ степени не выше $3 n-2$.

3. Функция $V_{n}^{\overline{0}}(\{u\})$ тождественно равна нулю, если $u_{\mu}^{2}=u_{\nu}^{2}$ для некоторого $\mu \neq \nu$.

Можно показать [6], [7], что функция, удовлетворяющая трем перечисленным выше свойствам, пропорциональна детерминанту матрицы

$$
P_{n}(\{u\})=\left(\begin{array}{ccccc}
u_{1}^{3 n-2} & u_{2}^{3 n-2} & u_{3}^{3 n-2} & \cdots & u_{2 n}^{3 n-2} \\
u_{1}^{3 n-4} & u_{2}^{3 n-4} & u_{3}^{3 n-4} & \cdots & u_{2 n}^{3 n-4} \\
u_{1}^{3 n-8} & u_{2}^{3 n-8} & u_{3}^{3 n-8} & \cdots & u_{2 n}^{3 n-8} \\
\vdots & \vdots & \vdots & \ddots & \vdots \\
u_{1}^{-3 n+2} & u_{2}^{-3 n+2} & u_{3}^{-3 n+2} & \cdots & u_{2 n}^{-3 n+2}
\end{array}\right) .
$$

Аналогично для функций $V_{n}^{\overline{1}}(\{u\})$ сформулируем следующие свойства.

1. Для каждого $\mu=1, \ldots, 2 n$ функция $V_{n}^{\overline{1}}(\{u\})$ удовлетворяет функциональному уравнению

$$
\begin{gathered}
V_{n}^{\overline{1}}\left(u_{1}, \ldots, u_{\mu}, \ldots, u_{2 n}\right)+V_{n}^{\overline{1}}\left(u_{1}, \ldots, a^{2} u_{\mu}, \ldots, u_{2 n}\right)+ \\
+V_{n}^{\overline{1}}\left(u_{1}, \ldots, a^{4} u_{\mu}, \ldots, u_{2 n}\right)=0 .
\end{gathered}
$$

2. Для каждого $\mu=1, \ldots, 2 n$ функция $u_{\mu}^{3 n-1} V_{n}^{\overline{1}}(\{u\})$ является полиномом по $u_{\mu}^{2}$ степени не выше $3 n-1$.

3. Функция $V_{n}^{\overline{1}}(\{u\})$ тождественно равна нулю, если $u_{\mu}^{2}=u_{\nu}^{2}$ для некоторого $\mu \neq \nu$.

Можно показать [15], что функция, удовлетворяющая этим трем свойствам, пропорциональна детерминанту матрицы

$$
Q_{n}(\{u\})=\left(\begin{array}{ccccc}
u_{1}^{3 n-1} & u_{2}^{3 n-1} & u_{3}^{3 n-1} & \cdots & u_{2 n}^{3 n-1} \\
u_{1}^{3 n-5} & u_{2}^{3 n-5} & u_{3}^{3 n-5} & \cdots & u_{2 n}^{3 n-5} \\
u_{1}^{3 n-7} & u_{2}^{3 n-7} & u_{3}^{3 n-7} & \cdots & u_{2 n}^{3 n-7} \\
\vdots & \vdots & \vdots & \ddots & \vdots \\
u_{1}^{-3 n+1} & u_{2}^{-3 n+1} & u_{3}^{-3 n+1} & \cdots & u_{2 n}^{-3 n+1}
\end{array}\right) .
$$

И, наконец, функция $V_{n}^{\overline{2}}(\{u\})$ удовлетворяет тем же свойствам, что и функция $V_{n}^{\overline{0}}(\{u\})$. Следовательно, она также пропорциональна детерминанту матрицы $P_{n}(\{u\})$. 
Мы не приводим здесь выражения для коэффициентов пропорциональности между функциями $V_{n}^{r}(\{u\})$ и детерминантами матриц $P(\{u\})$ и $Q(\{u\})$, а переходим непосредственно к рассмотрению частичных статистических сумм.

\section{4. СТАТИСТИЧЕСКИЕ СУММЫ}

Найдем явные выражения для частичных статистических сумм. Сначала заметим, что в общем эллиптическом случае частичные статистические суммы модели удовлетворяют некоторым рекуррентным соотоношениям [1], [11]. В частности, мы имеем

$$
\begin{aligned}
& \left.Z_{n}^{r}\left(\chi_{1}, \ldots, \chi_{n-1}, \chi_{n} ; \psi_{1}, \ldots, \psi_{n-1}, \psi_{n}\right)\right|_{\chi_{n}=\psi_{n}+\pi / 3}=\theta_{1}^{2-2 n}(2 \pi / 3) \times \\
& \quad \times \frac{\theta_{4}(\lambda+2 \pi(r+n) / 3)}{\theta_{4}(\lambda+2 \pi(r+n-1) / 3)} \prod_{i=1}^{n-1} \theta_{1}\left(\chi_{i}-\psi_{n}+\frac{\pi}{3}\right) \prod_{i=1}^{n-1} \theta_{1}\left(\psi_{n}-\psi_{i}+2 \pi / 3\right) \times \\
& \quad \times \widetilde{Z}_{n-1}^{r}\left(\chi_{1}, \ldots, \chi_{n-1} ; \psi_{1}, \ldots, \psi_{n-1}\right) .
\end{aligned}
$$

В тригонометрическом пределе эти соотношения принимают вид

$$
\begin{aligned}
& \left.Z_{n}^{r}\left(u_{1}, \ldots, u_{2 n-1}, u_{2 n}\right)\right|_{u_{2 n}=a^{-1} u_{2 n-1}}= \\
& \quad=a^{-2} \sigma^{2-2 n}\left(a^{2}\right) \frac{\sigma\left(a^{2(r+n)} b\right)}{\sigma\left(a^{2(r+n-1)} b\right)} \prod_{\mu=1}^{2 n-2} \sigma\left(a^{-1} u_{\mu} u_{2 n}^{-1}\right) Z_{n-1}^{r}\left(u_{1}, \ldots, u_{2 n-2}\right) .
\end{aligned}
$$

Удобно ввести функции

$$
Z_{n}^{\prime r}(\{u\})=\frac{a^{2(n-1)} \sigma^{n(n-1)}\left(a^{2}\right)}{\sigma\left(a^{2(n+r)} b\right)} Z_{n}^{r}(\{u\}),
$$

удовлетворяющие более простым рекуррентным соотношениям

$$
\begin{aligned}
& \left.Z_{n}^{\prime r}\left(u_{1}, \ldots, u_{2 n-2}, u_{2 n-1}, u_{2 n}\right)\right|_{u_{2 n}=a^{-1} u_{2 n-1}}= \\
& \quad=\prod_{\mu=1}^{2 n-2} \sigma\left(a^{-1} u_{\mu} u_{2 n-1}^{-1}\right) Z_{n-1}^{\prime r}\left(u_{1}, \ldots, u_{2 n-2}\right) .
\end{aligned}
$$

Учитывая, что частичные статистические суммы могут быть выражены через функции $V_{n}^{r}(\{u\})$, мы видим, что для функций $Z_{n}^{\prime r}(\{u\})$ справедливо представление

$$
Z_{n}^{\prime r}(\{u\})=A_{n}^{r} \mathcal{P}_{n}(\{u\})+B_{n}^{r} \prod_{i=1}^{n}\left(u_{2 i-1}^{-1} u_{2 i}\right) \mathcal{Q}_{n}(\{u\})+C_{n}^{r} \prod_{i=1}^{n}\left(u_{2 i-1}^{-2} u_{2 i}^{2}\right) \mathcal{P}_{n}(\{u\}),
$$

где

$$
\begin{aligned}
& \mathcal{P}_{n}(\{u\})=\frac{1}{\prod_{1 \leqslant \mu<\nu \leqslant 2 n} \sigma\left(u_{\mu} u_{\nu}^{-1}\right)} \operatorname{det} P_{n}(\{u\}), \\
& \mathcal{Q}_{n}(\{u\})=\frac{1}{\prod_{1 \leqslant \mu<\nu \leqslant 2 n} \sigma\left(u_{\mu} u_{\nu}^{-1}\right)} \operatorname{det} Q_{n}(\{u\}),
\end{aligned}
$$


а $A_{n}^{r}, B_{n}^{r}, C_{n}^{r}$ являются некоторыми постоянными. Заметим, что функции $\mathcal{P}_{n}(\{u\})$ и $\mathcal{Q}_{n}(\{u\})$ являются симметрическими функциями переменных $u_{1}, \ldots, u_{2 n}$. Фактически функции $\mathcal{P}_{n}(\{u\})$ непосредственно связаны с определенными функциями Шура:

$$
\mathcal{P}_{n}(\{u\})=\left(u_{1} \ldots u_{2 n}\right)^{-n} s_{(n-1, n-1, n-2, n-2, \ldots, 1,1,0,0)}\left(u_{1}^{2}, \ldots, u_{2 n}^{2}\right),
$$

что было замечено Окадой [13]. Для функций $\mathcal{Q}_{n}(\{u\})$ мы имеем аналогичное равенство:

$$
\mathcal{Q}_{n}(\{u\})=\left(u_{1} \ldots u_{2 n}\right)^{-n-1} s_{(n, n-1, n-1, n-2, \ldots, 2,1,1,0)}\left(u_{1}^{2}, \ldots, u_{2 n}^{2}\right) .
$$

Можно показать, что функции $\operatorname{det} P_{n}(\{u\})$ и $\operatorname{det} Q_{n}(\{u\})$ удовлетворяют рекуррентным соотношениям

$$
\begin{aligned}
& \left.\operatorname{det} P_{n}\left(u_{1}, \ldots, u_{2 n-1}, u_{2 n}\right)\right|_{u_{2 n}=a^{-1} u_{2 n-1}}= \\
& \quad=(-1)^{n-1} \sigma(a) \prod_{\mu=1}^{2 n-2} \sigma\left(u_{\mu}^{3} u_{2 n-1}^{-3}\right) \operatorname{det} P_{n-1}\left(u_{1}, \ldots, u_{2 n-2}\right),
\end{aligned}
$$

$\left.\operatorname{det} Q_{n}\left(u_{1}, \ldots, u_{2 n-1}, u_{2 n}\right)\right|_{u_{2 n}=a^{-1} u_{2 n-1}}=$

$$
=(-1)^{n-1} \sigma(a) \prod_{\mu=1}^{2 n-2} \sigma\left(u_{\mu}^{3} u_{2 n-1}^{-3}\right) \operatorname{det} Q_{n-1}\left(u_{1}, \ldots, u_{2 n-2}\right) \text {. }
$$

Доказательство первого соотношения приведено в работе [7]. Второе соотношение доказывается аналогично. Рекуррентные соотношения (18), (19) сводятся к рекуррентным соотношениям для функций $\mathcal{P}(\{u\})$ и $\mathcal{Q}(\{u\})$ вида

$$
\begin{aligned}
& \left.\mathcal{P}_{n}\left(u_{1}, \ldots, u_{2 n-2}, u_{2 n-1}, u_{2 n}\right)\right|_{u_{2 n}=a^{-1} u_{2 n-1}}= \\
& =(-1)^{n-1} \prod_{\mu=1}^{2 n-2} \sigma\left(a^{-1} u_{\mu} u_{2 n-1}^{-1}\right) \mathcal{P}_{n-1}\left(u_{1}, \ldots, u_{2 n-2}\right), \\
& \left.\mathcal{Q}_{n}\left(u_{1}, \ldots, u_{2 n-2}, u_{2 n-1}, u_{2 n}\right)\right|_{u_{2 n}=a^{-1} u_{2 n-1}}= \\
& =(-1)^{n-1} \prod_{\mu=1}^{2 n-2} \sigma\left(a^{-1} u_{\mu} u_{2 n-1}^{-1}\right) \mathcal{Q}_{n-1}\left(u_{1}, \ldots, u_{2 n-2}\right) .
\end{aligned}
$$

Используя представление (17) и рекуррентные соотношения (16), (20) и (21), мы получаем следующие рекуррентные соотношения для коэффициентов:

$$
A_{n}^{r}=(-1)^{n-1} A_{n-1}^{r}, \quad B_{n}^{r}=(-1)^{n-1} a B_{n-1}^{r}, \quad C_{n}^{r}=(-1)^{n-1} a^{2} C_{n-1}^{r} .
$$

При выводе этих рекуррентных соотношений мы предполагали, что три функции, входящие в правую часть равенства (17), линейно независимы. Легко видеть, что при $n=1$ это не так, и рекуррентные соотношения (22) в действительности верны лишь для $n>2$. В результате длинных вычислений находим

$$
A_{2}^{r}=-\frac{a^{4 r-2} b^{2}}{\sigma\left(b^{3}\right)}, \quad B_{2}^{r}=-\frac{1}{\sigma\left(b^{3}\right)}, \quad C_{2}^{r}=-\frac{a^{2-4 r} b^{-2}}{\sigma\left(b^{3}\right)} .
$$


Используя эти равенства как начальные данные для рекуррентных соотношений (22), получаем

$$
\begin{gathered}
A_{n}^{r}=(-1)^{n(n-1) / 2} \frac{a^{4 r-2} b^{2}}{\sigma\left(b^{3}\right)}, \quad B_{n}^{r}=(-1)^{n(n-1) / 2} \frac{a^{n-2}}{\sigma\left(b^{3}\right)}, \\
C_{n}^{r}=(-1)^{n(n-1) / 2} \frac{a^{2 n-4 r-2} b^{-2}}{\sigma\left(b^{3}\right)} .
\end{gathered}
$$

Отметим, что эти выражения справедливы для всех $n \geqslant 1$.

Таким образом, мы нашли явные выражения для частичных статистических сумм для статистической модели трех цветов в тригонометрическом пределе. В общем эллиптическом случае соответствующее выражение пока не найдено.

Благодарности. Авторы благодарят за гостеприимство Международный институт математической физики им. Эрвина Шредингера, где была выполнена основная часть настоящей работы. А. В. Разумов выражает благодарность Г. Боосу, $\Phi$. Гёману и А. Клюмперу за гостеприимство в университете г. Вуперталь и интересные обсуждения. Настоящая работа выполнена при частичной поддержке РФФИ (грант № 07-01-00234). А. В. Разумов был также частично поддержан совместным грантом РФФИ-ННИО (грант № 08-01-91953).

\section{Список литературы}

[1] А. В. Разумов, Ю. Г. Строганов, ТМФ, 161:1 (2009), 3-20; arXiv: 0805.0669.

[2] R. J. Baxter, J. Math. Phys., 11:10 (1970), 3116-3124.

[3] Р. Бэкстер, Точно решаемые модели в статистической механике, Мир, М., 1985.

[4] Ю.Г. Строганов, Общие свойства и частные решения уравнения треугольника. Вычисление статистической суммы для некоторых моделей на плоской решетке, Дис. ... канд. физ.-мат. наук, ИВФЭ, Протвино, 1982.

[5] Э.Т. Уиттекер, Дж.Н. Ватсон, Курс современного анализа, Гостехиздат, Л.-М., 1933-1934.

[6] Ю.Г. Строганов, ТMФ, 146:1 (2006), 65-76; arXiv: math-ph/0204042.

[7] А. В. Разумов, Ю. Г. Строганов, ТМФ, 141:3 (2004), 323-347; arXiv: math-ph/0312071.

[8] А. В. Разумов, Ю. Г. Строганов, ТМФ, 148:3 (2006), 357-386; arXiv: math-ph/0504022.

[9] А. В. Разумов, Ю. Г. Строганов, ТМФ, 149:3 (2006), 395-408; arXiv: math-ph/0507003.

[10] R. J. Baxter, Ann. Phys., 76:1 (1973), 25-47.

[11] H. Rosengren, Adv. Appl. Math., 43:2 (2009), 137-155; arXiv: 0801.1229.

[12] S. Okada, J. Algebra, 205:2 (1998), 337-367.

[13] S. Okada, J. Algebraic Combin., 23:1 (2006), 43-69; arXiv: math/0408234.

[14] A. Lenard, J. Math. Phys., 2:5 (1961), 682-693.

[15] Yu. G. Stroganov, Izergin-Korepin determinant reloaded, arXiv: math-ph/0409072.

Поступила в редакцию 26.03.2009 